\title{
NOTE ON THE TRANSFORMATION OF A GROUP INTO ITS CANONICAL FORM.
}

\author{
BY DR. S. H. SLOCUM.
}

(Read before the American Mathematical Society, December 28, 1901.)

LET

$$
X_{j} \equiv \sum_{1}^{n} \xi_{j k}\left(x_{1}, \cdots, x_{n}\right) \frac{\partial}{\partial x_{k}} \quad(j=1,2, \cdots, r)
$$

where the $\xi$ 's are analytic functions of $n$ independent variables $x_{1}, \ldots, x_{n}$ ), denote $r$ independent infinitesimal transformations of a given $r$ parameter group. The finite equations of the one-parameter groups generated by each of the infinitesimal transformations $X_{j}(j=1,2, \ldots, r)$ may be obtained by integration of the $r$ simultaneous systems

$$
\begin{gathered}
\frac{d x_{1}{ }^{\prime}}{\xi_{j 1}\left(x_{1}{ }^{\prime}, \cdots, x_{n}{ }^{\prime}\right)}=\cdots=\frac{d x_{n}{ }^{\prime}}{\xi_{j n}\left(x_{1}{ }^{\prime}, \cdots, x_{n}{ }^{\prime}\right)}=d a \\
(j=1,2, \cdots, r),
\end{gathered}
$$

subject to the condition that $x_{i}^{\prime}=x_{i}(i=1,2, \cdots, n)$ for $a=0, a$ being an arbitrary parameter. Let the integrals of these simultananeous systems be represented by the equations

$$
x_{i}^{\prime}=f_{i k}\left(x_{1}, \cdots, x_{n}, a\right) \quad(i=1,2, \cdots, n ; k=1,2, \cdots, r) .
$$

Performing upon the manifold $x_{1}, \cdots, x_{n}$ a general transformation $T_{1}$ of the one parameter group generated by $X_{1}$ we obtain the manifold $x_{1}^{\prime}, \cdots, x_{n}{ }^{\prime}$; performing upon this latter manifold a general transformation $T_{2}$ generated by $X_{2}$, we obtain the manifold $x_{1}^{\prime \prime}, \cdots, x_{n}{ }^{\prime \prime}$, etc. Thus we have

$$
\begin{aligned}
& x_{1}^{\prime}=f_{11}\left(x_{1}, \cdots, x_{n}, a_{1}\right), \quad \cdots x_{n}{ }^{\prime}=f_{n_{1}}\left(x_{1}, \cdots, x_{n}, a_{1}\right), \\
& x_{1}{ }^{\prime \prime}=f_{12}\left(x_{1}{ }^{\prime}, \cdots, x_{n}{ }^{\prime}, a_{2}\right), \quad \cdots x_{n}{ }^{\prime \prime}=f_{n_{2}}\left(x_{1}{ }^{\prime}, \cdots, x_{n}{ }^{\prime}, a_{2}\right) \text {, } \\
& x_{1}^{(r)}=f_{1 r}\left(x_{1}^{(r-1} \cdots, x_{n}^{(r-1)}, a_{r}\right), \cdots x_{n}^{(r)}=f_{n r}\left(x_{1}^{(r-1)}, \cdots, x_{n}^{(r-1)}, a_{r}\right),
\end{aligned}
$$

where $a_{1}, \cdots, a_{r}$ are arbitrary parameters. Eliminating $x_{1}^{\prime}, \cdots, x_{n}^{(r-1)}$ between these equations, we have

$$
x_{i}^{(r)}=f_{i}\left(x_{1}, \cdots, x_{n}, a_{1}, \cdots, a_{r}\right) \quad(i=1,2, \cdots, n),
$$


or, if we denote the transformed variables $x_{1}^{(r)}, \cdots, x_{n}^{(r)}$ by $x_{1}{ }^{\prime}, \cdots, x_{n}{ }^{\prime}$ respectively,

$$
x_{i}^{\prime}=f_{i}\left(x_{1}, \cdots, x_{n}, a_{1}, \cdots, a_{r}\right) \quad(i=1,2, \cdots, n),
$$

in which the parameters $a_{1}, \cdots, a_{r}$ are all essential. These equations define $\infty^{r}$ transformations of the given $r$-parameter group.*

This method will now be applied to finding the finite equations of the group whose infinitesimal transformations are

$$
X_{1} \equiv \frac{\partial}{\partial x_{2}}, \quad X_{2} \equiv x_{2} \frac{\partial}{\partial x_{2}}+\frac{\partial}{\partial x_{1}}
$$

The infinitesimal transformation $\frac{\partial}{\partial x_{2}}$ generates the one-parameter group whose finite equations are

$$
x_{1}^{\prime}=x_{1}, \quad x_{2}^{\prime}=x_{2}+a_{1},
$$

and similarly $x_{2} \frac{\partial}{\partial x_{2}}+\frac{\partial}{\partial x_{1}}$ generates the one-parameter group whose finite equations are

$$
x_{1}^{\prime \prime}=x_{1}^{\prime}+a_{2}, \quad x_{2}^{\prime \prime}=x_{2}^{\prime} e^{a_{2}} .
$$

Eliminating $x_{1}^{\prime}, x_{2}^{\prime}$ between equations (2) and (3) we have

$$
x_{1}^{\prime \prime}=x_{1}+a_{2}, \quad x_{2}^{\prime \prime}=x_{2} e^{a_{2}}+a_{1} e^{a_{2}}
$$

or, replacing $x_{1}^{\prime \prime}, x_{2}^{\prime \prime}$ by $x_{1}^{\prime}, x_{2}^{\prime}$,

$$
x_{1}^{\prime}=x_{1}+a_{2}, \quad x_{2}^{\prime}=x_{2} e^{a_{2}}+a_{1} e^{a_{2}} .
$$

These equations define a transformation $T_{a}$ of the given group $G$. Similarly, the equations defining a transformation $T_{b}$ of $G$ are

$$
x_{1}^{\prime \prime}=x_{1}^{\prime}+b_{2}, \quad x_{2}^{\prime \prime}=x_{2}^{\prime} e^{b_{2}}+b_{1} e^{b_{2}} .
$$

The transformation $T_{b} T_{a}$, obtained by the composition of the transformations $T_{a}$ and $T_{b}$ in the order named, is defined by the equations

(6) $\quad x_{1}^{\prime \prime}=x_{1}+a_{2}+b_{2}, \quad x_{2}^{\prime \prime}=x_{2} e^{a_{2}+b_{2}}+a_{1} e^{a_{2}+b_{2}}+b_{1} e^{b_{2}}$.

* Lie, Continuierliche Gruppen, pp. 192-197. 
If this is equivalent to a transformation $T_{c}$ of $G$, we also have

$$
x_{1}^{\prime \prime}=x_{1}+c_{2}, \quad x_{2}^{\prime \prime}=x_{2} e^{c_{2}}+c_{1} e^{c_{2}}
$$

Therefore

$$
\begin{aligned}
& c_{1}=a_{1}+b_{1} e^{-a_{2}} \equiv \varphi_{1}(a, b), \\
& c_{2}=a_{2}+b_{2} \equiv \varphi_{2}(a, b) .
\end{aligned}
$$

The c's are finite for every finite system of values of the $a$ 's and 'b's, but the transformation $T_{b} T_{a}$, or $T_{c}$, may not be generated by an infinitesimal transformation of the group, as will appear later.

In order to transform the finite equations

$$
x_{i}^{\prime}=f_{i}\left(x_{1}, \cdots, x_{n}, a_{1}, \cdots, a_{r}\right) \quad(i=1,2, \cdots, n)
$$

of the group into their canonical form, Lie proceeds as follows.* By differentiation and elimination we obtain the differential equations

$$
\begin{gathered}
\frac{\partial x_{i}^{\prime}}{\partial a_{k}}=\sum_{1}^{r} \psi_{j k}\left(a_{1}, \cdots, a_{r}\right) \xi_{j i}\left(x_{1}{ }^{\prime}, \cdots, x^{\prime}{ }_{n}\right) \\
(i=1,2, \cdots, n ; k=1,2, \cdots, r),
\end{gathered}
$$

which are satisfied identically by equations (1) above. Since the determinant of the $\psi_{j k} \neq 0$, these equations may be written in the form

$$
\begin{gathered}
\xi_{j i}\left(x_{1}{ }^{\prime}, \cdots, x_{n}{ }^{\prime}\right)=\sum_{1}^{r} \alpha_{j k}\left(a_{1}, \cdots, a_{r}\right) \frac{\partial x_{i}^{\prime}}{\partial a_{k}} \\
(i=1,2, \cdots, n ; j=1,2, \cdots, r),
\end{gathered}
$$

where the determinant of the $\alpha_{j k}(\alpha) \neq 0$, and no linear relation of the form $e_{1} \xi_{1 i}\left(x^{\prime}\right)+\cdots+e_{r} \xi_{r i}\left(x^{\prime}\right) \equiv 0$, with constant coefficients $e$ persists simultaneously for $i=1,2, \cdots, n$. The canonical form of the finite equations of the group can now be obtained by integration of the simultaneous system

$$
\frac{d x_{1}^{\prime}}{\sum_{1}^{r} \lambda_{j} \xi_{j 1}\left(x^{\prime}\right)}=\cdots=\frac{d x_{n}^{\prime}}{\sum_{1}^{r} \lambda_{j} \xi_{j n}\left(x^{\prime}\right)}=d t,
$$

\footnotetext{
* Transformationsgruppen, vol. 3 , pp. 609-611.
} 
that is to say, by integration of the simultaneous system

$$
\frac{d x_{i}^{\prime}}{d t}=\sum_{k, j}^{1 \cdots r} \lambda_{j} a_{j k}\left(a_{1}, \cdots, a_{r}\right) \frac{\partial x_{i}^{\prime}}{\partial a_{k}} \quad(i=1,2, \cdots, n),
$$

subject to the condition that $x_{i}^{\prime}=x_{i}(i=1,2, \cdots, n)$ for $t=0$. Consequently $a_{1}, \cdots, a_{r}$, considered as functions of $t$, satisfy the simultaneous system

$$
\frac{d \alpha_{k}}{d t}=\sum_{1}^{r} \lambda_{j} \alpha_{j k}\left(\alpha_{1}, \cdots, \alpha_{r}\right) \quad(k=1,2, \cdots, r) .
$$

Since $x_{i}^{\prime}=x_{i}(i=1,2, \cdots, n)$ for $t=0, a_{1}, \cdots, a_{r}$ must assume the values $a_{1}^{0}, \cdots, a_{r}^{0}$ for $t=0$, where $a_{1}^{0}, \cdots, a_{r}^{0}$ are the parameters which furnish the identical transformation. Integrating the simultaneous system (9) subject to the condition that $a_{k}=a_{k}^{0}(k=1,2, \cdots, r)$ for $t=0$, we obtain the integrals

$$
a_{k}=M_{k}\left(\lambda_{1} t, \cdots, \lambda_{r} t\right) \quad(k=1,2, \cdots, r),
$$

or, if we denote the parameters $\lambda_{1} t, \cdots, \lambda_{r} t$ by $\mu_{1}, \cdots, \mu_{r}$, respectively, we have

$$
a_{k}=M_{k}\left(\mu_{1}, \cdots, \mu_{r}\right) \quad(k=1,2, \cdots, r) .
$$

Inserting these values of $a_{1}, \cdots, a_{r}$ in equations (1), we have

$$
x_{i}^{\prime}=f_{i}\left(x_{1}, \cdots, x_{n}, M_{1}(\mu), \cdots, M_{r}(\mu)\right) \quad(i=1,2, \cdots, n),
$$

which are the canonical equations of the given group.

Consider the application of this method to the finite equations (4) on page 281 . Since the equations

$$
\begin{aligned}
& x_{1}^{\prime}=x_{1}+a_{2} \equiv f_{1}(x, a), \\
& x_{2}^{\prime}=x_{2} e^{a_{2}}+a_{1} e^{a_{2}} \equiv f_{2}(x, a)
\end{aligned}
$$

define $\infty^{2}$ transformations $T_{a}$ which constitute a group, functional equations persist of the form

or

$$
\begin{aligned}
f_{i}(f(x, a), b) & =f_{i}(x, \varphi(a, b)) & (i=1,2), \\
f_{i}\left(x^{\prime}, b\right) & =f_{i}(x, c) & (i=1,2) .
\end{aligned}
$$

The functions $\varphi_{1}, \varphi_{2}$ in equations (8) are independent of one another with respect to $b_{1}, b_{2}$, for

$$
\frac{\partial \varphi_{h}}{\partial b_{j}}=\left|\begin{array}{cc}
e^{-a_{2}}, & 0 \\
0, & 1
\end{array}\right|
$$


is not identically zero. Therefore we may regard $x_{1}, x_{2}, a_{1}$, $a_{2}, c_{1}, c_{2}$ as independent variables, and $x_{1}^{\prime}, x_{2}{ }^{\prime}, x_{1}{ }^{\prime \prime}, x_{2}{ }^{\prime \prime}, b_{1}, b_{2}$ as dependent variables. Thus the differentiation of the functional equations (10), that is, of

$$
\begin{aligned}
x_{1}^{\prime}+b_{2} & =x_{1}+c_{2}, \\
x_{2}^{\prime} e^{b_{2}}+b_{1} e^{b_{2}} & =x_{2} e^{c_{2}}+c_{1} e^{c_{2}},
\end{aligned}
$$

with respect to the $a^{\prime}$ s gives

$$
\frac{\partial x_{1}^{\prime}}{\partial a_{1}}+\frac{\partial b_{2}}{\partial a_{1}}=0, \quad \frac{\partial x_{1}^{\prime}}{\partial a_{2}}+\frac{\partial b_{2}}{\partial a_{2}}=0
$$

$$
\begin{aligned}
& e^{b_{2}} \frac{\partial x_{2}^{\prime}}{\partial a_{1}}+\left(x_{2}^{\prime}+b_{1}\right) e^{b_{2}} \frac{\partial b_{2}}{\partial a_{1}}+e^{b_{2}} \frac{\partial b_{1}}{\partial a_{1}}=0, \\
& e^{b_{2}} \frac{\partial x_{2}^{\prime}}{\partial a_{2}}+\left(x_{2}^{\prime}+b_{1}\right) e^{b_{2}} \frac{\partial b_{2}}{\partial a_{2}}+e^{b_{2}} \frac{\partial b_{1}}{\partial a_{2}}=0 .
\end{aligned}
$$

In order to obtain expressions for $\frac{\partial b_{j}}{\partial a_{k}}$, we differentiate equations (8) with respect to $a_{1}, a_{2}$, and thus obtain

whence

$$
\begin{aligned}
0=\frac{\partial b_{2}}{\partial a_{1}}, & 0=1+\frac{\partial b_{2}}{\partial a_{2}}, \quad 0=1+e^{-a_{2}} \frac{\partial b_{1}}{\partial a_{1}}, \\
0 & =-b_{1} e^{-a_{2}}+e^{-a_{2}} \frac{\partial b_{1}}{\partial a_{2}},
\end{aligned}
$$

$$
\frac{\partial b_{1}}{\partial a_{1}}=-e^{a_{2}}, \quad \frac{\partial b_{1}}{\partial a_{2}}=b_{1}, \quad \frac{\partial b_{2}}{\partial a_{i}}=0, \quad \frac{\partial b_{2}}{\partial a_{2}}=-1 .
$$

Inserting these values, equations (11) become

$$
\frac{\partial x_{1}^{\prime}}{\partial a_{1}}=0, \quad \frac{\partial x_{1}^{\prime}}{\partial a_{2}}=1, \quad \frac{\partial x_{2}^{\prime}}{\partial a_{1}}=e^{a_{2}}, \quad \frac{\partial x_{2}^{\prime}}{\partial a_{2}}=x_{2}^{\prime} .
$$

These equations are of the form

$$
\begin{gathered}
\frac{\partial x_{i}^{\prime}}{\partial a_{k}}=\Psi_{1 k}(a, b) \Phi_{1 i}\left(x^{\prime}, b\right)+\Psi_{2 k}(a, b) \Phi_{2 i}\left(x^{\prime}, b\right) \\
(i=1,2 ; k=1,2),
\end{gathered}
$$


where

Therefore

$$
\Psi_{i k}(a, b) \equiv \frac{\partial b_{i}}{\partial a_{k}} \quad(i=1,2 ; k=1,2)
$$

$$
\Psi_{11}=-e^{a_{2}}, \quad \Psi_{12}=b_{1}, \quad \Psi_{21}=0, \quad \Psi_{22}=-1,
$$

and, consequently,

$$
\Phi_{11}=0, \quad \Phi_{12}=-1, \quad \Phi_{21}=-1, \quad \Phi_{22}=-x_{2}{ }^{\prime}-b_{1} .
$$

If we insert in equations (12) the values of $x_{1}^{\prime}, x_{2}{ }^{\prime}$ derived from

$$
x_{2}^{\prime}=x_{2} e^{a_{2}}+a_{1} e^{a_{2}}, \quad x_{1}^{\prime}=x_{1}+a_{2} \text {, }
$$

they become equations between the independent quantities $x_{1}, x_{2}, a_{1}, a_{2}, b_{1}, b_{2}$, and must therefore be satisfied identically. Hence equations (12) will still persist identically in virtue of equations (4) if we assign definite values $\vec{b}_{1}, \bar{b}_{2}$ to $b_{1}, b_{2}$. For this purpose let $b_{1}=1$, and denote the functions $\Psi(a, \bar{b})$ and $\Phi\left(x^{\prime}, \bar{b}\right)$ by $\psi(a)$ and $\xi\left(x^{\prime}\right)$ respectively. Then we have

where

$$
\begin{gathered}
\frac{\partial x_{i}^{\prime}}{\partial a_{k}}=\psi_{1 k}(a) \xi_{1 i}\left(x^{\prime}\right)+\psi_{2 k}(a) \xi_{2 i}\left(x^{\prime}\right) \\
(i=1,2 ; k=1,2),
\end{gathered}
$$

$$
\begin{aligned}
& \xi_{11}=0, \quad \xi_{12}=-1, \quad \xi_{21}=-1, \quad \xi_{22}=-x_{2}^{\prime}-1, \\
& \psi_{11}=-e^{a_{2}}, \quad \psi_{12}^{\prime}=1, \quad \psi_{21}=0, \quad \psi_{22}=-1 .
\end{aligned}
$$

The determinant of the $\psi$ 's, namely

$$
\left|\begin{array}{ll}
\psi_{11}, & \psi_{12} \\
\psi_{21}, & \psi_{22}
\end{array}\right|=\left|\begin{array}{rr}
-e^{a_{2}}, & 1 \\
0, & -1
\end{array}\right|
$$

not being identically zero, equations (13) may be solved for the $\xi$ 's, giving

where

$$
\begin{gathered}
\xi_{j i}\left(x_{1}^{\prime}, x_{\imath}^{\prime}\right)=\alpha_{j 1}(a) \frac{\partial x_{i}^{\prime}}{\partial \alpha_{1}}+\alpha_{j 3}(a) \frac{\partial x_{i}^{\prime}}{\partial \alpha_{2}} \\
(i=1,2 ; j=1,2),
\end{gathered}
$$

$$
\alpha_{11}=-e^{-a_{2}}, \quad \alpha_{12}=0, \quad \alpha_{12}=-e^{-a_{2}}, \quad \alpha_{22}=-1 .
$$


In order to obtain the system of functions $a_{k}=M_{k}\left(\mu_{1}, \mu_{2}\right)$ $(k=1,2)$ which, when introduced into the finite equations (4) of the group, will transform these equations into their canonical form, it is necessary to integrate the simultaneous system

$$
\frac{d a_{k}}{d t}=\sum_{1}^{2} \lambda_{j} \alpha_{j k}\left(a_{1}, a_{2}\right) \quad(k=1,2)
$$

subject to the condition that $a_{k}=a_{k}^{0}(k=1,2)$ for $t=0$, that is, we must integrate the simultaneous system

$$
\frac{d a_{1}}{d t}=-\lambda_{1} e^{-a_{2}}-\lambda_{2} e^{-a_{2}}, \quad \frac{d a_{2}}{d t}=-\lambda_{2},
$$

subject to the condition that $a_{1}=a_{2}=0$ for $t=0$. The integrals of these equations are

$$
\begin{aligned}
& a_{1}=-\frac{\lambda_{1} t+\lambda_{2} t}{\lambda_{2} t}\left(e^{\lambda_{2} t}-1\right) \equiv M_{1}\left(\lambda_{1} t, \lambda_{2} t\right), \\
& a_{2}=-\lambda_{2} t \equiv M_{2}\left(\lambda_{1} t, \lambda_{2} t\right),
\end{aligned}
$$

or, if we let $\mu_{1}=-\left(\lambda_{1}+\lambda_{2}\right) t, \mu_{2}=-\lambda_{2} t$,

$$
\begin{aligned}
& a_{1}=-\frac{\mu_{1}}{\mu_{2}}\left(e^{-\mu_{2}}-1\right) \equiv M_{1}\left(\mu_{1}, \mu_{2}\right), \\
& a_{2}=\mu_{2} \equiv M_{2}\left(\mu_{1}, \mu_{2}\right) .
\end{aligned}
$$

Inserting these values, equations (4) become

$$
\begin{aligned}
& x_{1}^{\prime}=x_{1}+\mu_{2}, \\
& x_{2}^{\prime}=x_{2} e^{\mu_{2}}+\frac{\mu_{1}}{\mu_{2}}\left(e^{\mu_{2}}-1\right),
\end{aligned}
$$

which is the canonical form of the equations defining a transformation $T_{\mu}$ of the group. These equations are of precisely the same form as the equations obtained by summation of the series where

$$
x_{i}^{\prime}=x_{i}+\sum_{i}^{2} j_{j} X_{j} x_{i}+\frac{1}{2 !} \sum_{1}^{2} \sum_{1}^{2} k^{\prime \prime} j_{j} \mu_{k} X_{j} X_{k} x_{i}+\cdots \quad(i=1,2),
$$

$$
X_{1} \equiv \frac{\partial}{\partial x_{2}}, \quad X_{2} \equiv x_{2} \frac{\partial}{\partial x_{2}}+\frac{\partial}{\partial x_{1}}
$$

For every finite system of values of the $\mu$ 's, the $a$ 's are finite. Consequently every transformation of the family 
$T_{\mu}$ belongs to the family $T_{a}$, and is generated by an infinitesimal transformation of the group. Since $M_{1}(\mu), M_{2}(\mu)$ in equations (14) are independent functions of the $\mu$ 's, Lie assumes that every transformation of the family $T_{\alpha}$ belongs to the family $T_{\mu}$. But although the functions $a_{1}, a_{2}$, defined by equations (14), are independent of the $\mu$ 's since the Jacobian

$$
\frac{\partial\left(\alpha_{1}, \alpha_{2}\right)}{\partial\left(\mu_{1}, \mu_{2}\right)} \equiv \frac{e^{\mu_{2}}-1}{\mu_{2} e^{\mu_{2}}}
$$

is not identically zero, nevertheless for certain values of the $a$ 's the $\mu$ 's are infinite. Thus, solving equations (14), we have

$$
\mu_{1}=\frac{a_{1} a_{2} e^{a_{2}}}{e^{a_{2}}-1} \equiv N_{1}\left(a_{1}, a_{2}\right), \quad \mu_{2}=a_{2} \equiv N_{2}\left(a_{1}, a_{2}\right) .
$$

For $a_{1} \neq 0$ and $a_{2}$ an even multiple of $\pi \sqrt{-1}, \mu_{1}$ becomes infinite, and this transformation of the family $T_{a}$ is distinct from any transformation of the family $T_{a}$ for which the $\mu$ 's are finite. But infinite values of the $\mu$ 's are excluded from consideration, for $\mu_{1}=-\left(\lambda_{1}+\lambda_{2}\right) t, \mu_{2}=-\lambda_{2} t$, and since $t$ cannot be infinite if $\mu_{1}$ or $\mu_{2}$ is infinite, $\lambda_{1}$ or $\lambda_{2}$ must be infinite, and by supposition the $\lambda$ 's are arbitrary but definite constants. Consequently we cannot assume that every transformation of the family $T_{a}$ belongs to the family $T_{\mu}$.

This necessitates a restriction upon the criterion for the continuity of a group. For a system of values of the $a$ 's for which one or both of the functions $N_{1}(a), N_{2}(a)$ are infinite there is no equivalent transformation of the family $T_{\mu}$, and consequently such a transformation $T_{a}$ cannot be generated by an infinitesimal transformation of the group. For example, the transformation considered above, for which $a_{1} \neq 0, a_{2}=$ an even multiple of $\pi \sqrt{-1}$, cannot be generated by an infinitesimal transformation of the grcup. Such a transformation is termed a singular transformation, and a group which contains a singular transformation is said to be discontinuous.

A group is said to be continuous if it contains no singular transformation. In other words, an $r$-parameter group is said to be continuous if the composition of two arbitrary transformations $T_{a}$ and $T_{b}$ of the group, generated by the infinitesimal transformations

$$
a_{1} X_{1}+\cdots+a_{r} X_{r}, \quad b_{1} X_{1}+\cdots+b_{r} X_{r}
$$


respectively, is equivalent to a transformation $T_{c}$ of the group, generated by the infinitesimal transformation

$$
c_{1} X_{1}+\cdots+c_{r} X_{r}
$$

with finite parameters $c_{1}, \cdots, c_{r}$; that is to say, if a system of finite values of the $c$ 's can be found to satisfy the symbolic equation $T_{b} T_{a}=T_{c}$. On page 282 we saw that the composition of the two arbitrary transformations $T_{a}$ and $T_{b}$ of the family defined by equations (4) was equivalent to a transformation $T_{0}$ of the family, with finite parameters $c$. But equations (4) were not in their canonical form, and therefore it did not necessarily follow that the transformation $T_{c}$ could be generated by an infinitesimal transformation of the group, as shown above. Consequently, if the finite equations of a group are not in their canonical form, the condition that for every finite system of values of the $a$ 's and $b$ 's a finite system of the $c$ 's can be found to satisfy the symbolic equation $T_{b} T_{a}=T_{c}$ is a necessary but not a sufficient condition for the continuity of the group.

University of Cincinnati, December, 1901.

\section{SOME APPLICATIONS OF GREEN'S THEOREM IN ONE DIMENSION.}

BY MR. OTTO DUNKKL.

(Read before the American Mathematical Society, February 22, 1902.)

Green's theorem ordinarily has reference to Laplace's equation in either two or three dimensions. It has been generalized however in the case of two dimensions by replacing Laplace's equation by the general homogeneous linear differential equation of the second order. In the generalized form the theorem relates not only to the given differential equation, but also to its adjoint differential equation.* A further extension of the theorem is possible by considering a differential equation of the $n$th order in two or more independent variables, and its corresponding adjoint. $\dagger$

* Cf. Encyklopädie, II, A. 7 c., p. 513.

$\dagger$ Cf. Darboux, Théorie des Surfaces, vol. 2, pp. 72, 74, for the case of two independent variables. 